\title{
BFS Solution for Disjoint Paths in P Systems
}

\author{
Radu Nicolescu and Huiling Wu \\ Department of Computer Science, University of Auckland, \\ Private Bag 92019, Auckland, New Zealand \\ r.nicolescu@auckland.ac.nz, hwu065@aucklanduni.ac.nz
}

Summary. This paper continues the research on determining a maximum cardinality set of edge- and node-disjoint paths between a source cell and a target cell in P systems. We review the previous solution [3], based on depth-first search (DFS), and we propose a faster solution, based on breadth-first search (BFS), which leverages the parallel and distributed characteristics of $\mathrm{P}$ systems. The runtime complexity shows that, our BFSbased solution performs better than the DFS-based solution, in terms of P steps.

\section{Introduction}

P systems is a bio-inspired computational model, based on the way in which chemicals interact and cross cell membranes, introduced by Păun [16]. The essential specification of a $\mathrm{P}$ system includes a membrane structure, objects and rules. All cells evolve synchronously by applying rules in a non-deterministic and (potentially maximally) parallel manner. Thus, $\mathrm{P}$ systems is a strong candidate as a model for distributed and parallel computing.

Given a digraph $\mathrm{G}$ and two nodes, $s$ and $t$, the disjoint paths problem aims to find the maximum number of $s$-to- $t$ edge- or node-disjoint paths. There are many important applications that need to find alternative paths between two nodes, in all domains. Alternative paths are fundamental in biological remodelling, e.g., of nervous or vascular systems. Multipath routing can use all available bandwidth in computer networks. Disjoint paths are sought in streaming multi-core applications that are bandwidth sensitive to avoid sharing communication links between processors [17]. The maximum matching problem in a bipartite graph can also be transformed to the disjoint paths problem. In case of non-complete graphs, Byzantine Agreement requires at least $2 k+1$ node-disjoint paths, between each pair of nodes to ensure that a distributed consensus can occur, with up to $k$ failures [9].

It is interesting to design a native $\mathrm{P}$ system solution for the disjoint path problem. In this case, the input graph is the P system structure itself, not as data to a program. Also, the system is fully distributed, i.e. there is no central node and only local channels (between structural neighbours) are allowed. In 2010, Dinneen, 
Kim and Nicolescu [3] proposed the first P solution, as a distributed version of the Ford-Fulkerson algorithm, based on depth-first search (DFS). This solution searches by visiting nodes sequentially, which is not always efficient. To exploit the parallel potential of $\mathrm{P}$ systems, we propose a faster $\mathrm{P}$ system solution - $\mathrm{a}$ distributed version of the Edmonds-Karp algorithm, which concurrently searches as many paths as possible in breadth-first search (BFS).

This paper is organized as follows. Section 2 defines a simplified P system, general enough to cover most basic families. Section 3 describes the disjoint paths problem and the strategies for finding disjoint paths in digraphs. Section 4 discusses the specifics of the disjoint paths problem in $\mathrm{P}$ systems. Section 5 reviews the previous DFS-based solution [3] and sets out our faster BFS-based solution. Section 6 presents the $\mathrm{P}$ system rules for the disjoint paths algorithm using BFS. Section 7 compares the performance of the BFS-based and DFS-based algorithms, in terms of $\mathrm{P}$ steps, and the relative performance of the BFS-based solution simulation on sequential vs. parallel (multi-core) hardware. Finally, Section 8 summarizes our work and highlights future work.

\section{Preliminary}

Essentially, a static $\mathrm{P}$ system is specified by the membrane structure, objects and rules. The membrane structure can be modeled as: a rooted tree (cell-like P systems [16]), a directed acyclic graph (hyperdag P systems [11], [12], [13]), or in a more general case, an arbitrary digraph (neural P systems [10], [14]). Usually, the objects are symbols from a given alphabet, but one can also consider strings or other more complex structures (such as tuples). P systems combine rewriting rules that change objects in the region and communication rules that move objects across membranes. Here, we define a simple $\mathrm{P}$ system, with priorities, promoters and duplex channels as a system, $\Pi=\left(O, \sigma_{1}, \sigma_{2}, \ldots, \sigma_{n}, \delta\right)$, where:

1. $O$ is a finite non-empty alphabet of objects;

2. $\sigma_{1}, \ldots, \sigma_{n}$ are cells, of the form $\sigma_{i}=\left(Q_{i}, s_{i, 0}, w_{i, 0}, R_{i}\right), 1 \leq i \leq n$, where:

- $Q_{i}$ is a finite set of states;

- $s_{i, 0} \in Q_{i}$ is the initial state;

- $w_{i, 0} \in O^{*}$ is the initial multiset of objects;

- $R_{i}$ is a finite ordered set of rewriting/communication rules of the form: $\left.s x \rightarrow_{\alpha} s^{\prime} x^{\prime}(y)_{\beta}\right|_{z}$, where: $s, s^{\prime} \in Q_{i}, x, x^{\prime}, y, z \in O^{*}, \alpha \in\{\min , \max \}$, $\beta \in\{\uparrow, \downarrow, \uparrow\}$.

3. $\delta$ is a set of digraph $\operatorname{arcs}$ on $\{1,2, \ldots, n\}$, without symmetric arcs, representing duplex channels between cells.

The membrane structure is a digraph with duplex channels, so parents can send messages to children and children to parents, but the disjoint paths strictly follow the parent-child direction. Rules are prioritized and are applied in weak priority order [15]. 
The general form of a rule, which transforms state $s$ to state $s^{\prime}$, is $s x \rightarrow_{\alpha}$ $\left.s^{\prime} x^{\prime}(y)_{\beta_{\gamma}}\right|_{z}$. This rule consumes multiset $x$, and then (after all applicable rules have consumed their left-hand objects) produces multiset $x^{\prime}$, in the same cell ("here"). Also, it produces multiset $y$ and sends it, by replication, to all parents ("up"), to all children ("down"), or to all parents and children ("up and down"), according to the value of target indicator $\beta \in\{\uparrow, \downarrow, \uparrow\}$ (effectively, here we use the repl communication mode, exclusively). $\alpha \in\{\min , \max \}$ describes the rewriting mode. In the minimal mode, an applicable rule is applied exactly once. In the maximal mode, an applicable rule is used as many times as possible and all rules with the same states $s$ and $s^{\prime}$ can be applied in the maximally parallel manner. Finally, the optional $z$ indicates a multiset of promoters, which are not consumed, but are required, when determining whether the rule can be applied.

\section{Disjoint Paths}

Given a digraph, $G=(V, E)$, a source node, $s \in V$, and a target node, $t \in V$, the edge- and node-disjoint paths problem looks for one of the largest sets of edge- and node-disjoint $s$-to-t paths. A set of paths is edge-disjoint or node-disjoint if they have no common arc or no common intermediate node. Note that node-disjoint paths are also edge-disjoint paths, but the converse is not true. Cormen et al. [1] give a more detailed presentation of the topics discussed in this section.

Figure 1 (a) shows two node-disjoint paths from 0 to 6, i.e. 0.3.6 and 0.1.4.6, which are also edge-disjoint. In this scenario, this is the maximum number of node-disjoint paths one can find. However, one could add to this set another path, 0.2.3.5.6, shown in Figure 1 (b), to obtain a set of three edge-disjoint paths.
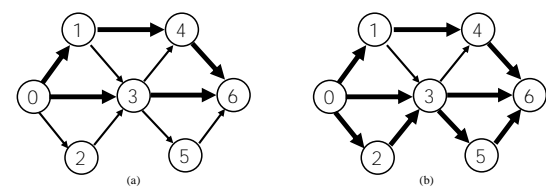

Fig. 1. Node- and edge- disjoint paths.

The maximum edge-disjoint paths problem can be transformed to a maximum flow problem by assigning unit capacity to each edge [5]. Given a set of already established edge- or node-disjoint paths $P$, we recall the definition of the residual digraph $G_{r}=\left(V_{r}, E_{r}\right)$ :

- $V_{r}=\mathrm{V}$ and

- $E_{r}=\left(E \backslash E_{P}\right) \cup E_{P}^{\prime}$, where $E_{P}$ is the set of $\operatorname{arcs}(u, v)$ that appear in the $P$ paths and $E_{P}^{\prime}=\left\{(v, u) \mid(u, v) \in E_{P}\right\}$.

Briefly, the residual digraph is constructed by reversing the already established path arcs. An augmenting path is an $s$-to- $t$ path in the residual digraph, $G_{r}$. 
Augmenting paths are used to extend the existing set of established disjoint paths. If an augmenting arc reverses an existing path arc (also known as a push-back operation), then these two arcs "cancel" each other, due to zero total flow, and are discarded. The remaining path fragments are relinked to construct an extended set of disjoint paths. This round is repeated, starting with the new and larger set of established paths, until no more augmenting paths are found. A more detailed construction appears in Ford and Fulkerson maximal flow algorithm [5].

Example 1. Figure 2 illustrates a residual digraph and an augmenting path: (a) shows a digraph, where two edge-disjoint paths, 0.1.4.7 and 0.2.5.7, are present; (b) shows the residual digraph, formed by reversing path arcs; (c) shows an augmenting path, 0.3.5.2.6.7, which uses a reverse arc, $(5,2)$; (d) discards the cancelling arcs, $(2,5)$ and $(5,2) ;(\mathrm{e})$ relinks the remaining path fragments, 0.1.4.7, 0.2, 5.7, 0.3.5 and 2.6.7, resulting in now three edge-disjoint paths, 0.1.4.7, 0.2.6.7 and 0.3.5.7.
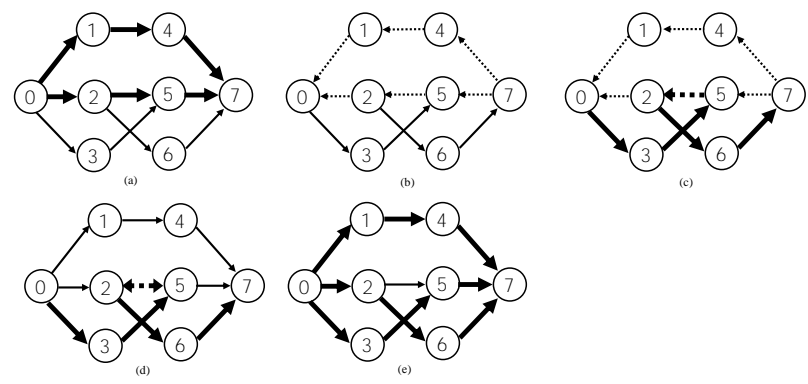

Fig. 2. Finding an augmenting path in the residual digraph.

The search for augmenting paths uses a search algorithm such as DFS (e.g., the Ford-Fulkerson algorithm) or BFS (e.g., the Edmonds-Karp algorithm). A search path in the residual graph (also known as a tentative augmenting path) starts from the source node and tries to reach the target node. A successful search path becomes a new augmenting path and is used (as previously explained) to increase the number of disjoint paths. Conceptually, this solves the edge-disjoint paths problem (at a high level). However, the node-disjoint paths require additional refinements - usually by node splitting [8]. Each intermediate node, $v$, is split into an entry node, $v_{1}$, and an exit node, $v_{2}$, linked by an $\operatorname{arc}\left(v_{1}, v_{2}\right)$. Arcs that in the original digraph, $G$, were directed into $v$ are redirected into $v_{1}$ and arcs that were directed out of $v$ are redirected out of $v_{2}$. Figure 3 illustrates this node-splitting procedure: (a) shows the original digraph and (b) the modified digraph, where all intermediate nodes are split - this is a bipartite digraph. 


\section{Disjoint Paths in P Systems}

Classical algorithms use the digraph as data and keep global information. In contrast, our solutions are fully distributed. There is no central cell to convey global information among all cells, i.e. cells only communicate with their neighbors via local channels (between structural neighbours).

Unlike traditional programs, which keep full path information globally, our $\mathrm{P}$ systems solution records paths predecessors and successors locally in each cell, similar to distributed routing tables in computer networks. To construct such routing indicators, we assume that each cell $\sigma_{i}$ is "blessed" with a unique cell ID object, $\iota_{i}$, functioning as a promoter.

Although many versions of $\mathrm{P}$ systems accept cell division, we feel that this feature should not be used here and we intentionally discard it. Rather than actually splitting the intermediate $\mathrm{P}$ cells, we simulate this by ad-hoc cell rules. This approach could be in other distributed networks, where nodes cannot be split [3]. Essentially, node splitting prevents more than one unit flow to pass through an intermediate node [8].

In our case, node splitting can be simulated by: (i) constraining in and out flow capacities to one and (ii) having two visited markers for each cell, one for a virtual entry node and another for a virtual exit node, extending the visiting idea of classical search algorithms. Figure 3 illustrates a scenario when one cell, $y$, is visited twice, first on its entry and then on its exit node [3]. Assume that path $\pi=$ s.x.y.z.t, is established. Consider a search path, $\tau$, starting from cell, $s$, and reaching cell, $y$, in fact, $y$ 's entry node. This is allowed and $y$ 's entry node is marked as visited. However, to constrain its in-flow to one, $y$ can only pushback $\tau$ on its in-flow arc, $(x, y)$. Cell $x$ 's exit node becomes visited, $x$ 's out-flow becomes zero and $\tau$ continues on $x$ 's outgoing arc, $(x, z)$. When $\tau$ reaches cell $z, z$ 's entry node becomes visited and $z$ pushes $\tau$ back on its in-flow arc, $(y, z)$. Cell $y$ 's exit node becomes visited, $y$ 's out-flow becomes zero and $\tau$ continues on $y$ 's outgoing arc, $(y, t)$. When no other outgoing arc is present, the cell needs to push-back from its exit node to its entry node, which is only possible if its entry node is not visited. Finally, the search path, $\tau$, reaches the target, $t$, and becomes $\tau=s . y . x . z . t$. After removing cancelling arcs and relinking the remaining ones, we have two node-disjoint paths, s.x.z.t and s.y.t.

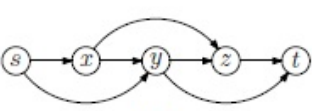

(a)

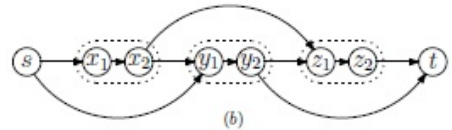

(b)

Fig. 3. Simulating node splitting [3]. 


\section{Distributed DFS-based and BFS-based Solutions}

As mentioned in Section 3, augmenting paths can be searched using DFS or BFS. Conceptually, DFS explores as far as possible along a single branch, before backtracking, while BFS explores as many branches as possible concurrently $-\mathrm{P}$ systems can exploit this parallelism.

\subsection{Distributed DFS-based Strategy}

Dinneen et al's DFS-based algorithms find disjoint paths in successive rounds [3].

Each round starts with a set of already established disjoint paths, which is empty at the start of the first round. The source cell, $\sigma_{s}$, starts to explore one of the untried branches. If the search path reaches the target cell, $\sigma_{t}$, it confirms to $\sigma_{s}$ that a new augmenting paths was found; otherwise, it backtracks. While moving towards $\sigma_{s}$, the confirmation reshapes the existing paths and the newly found augmenting path, i.e. discarding cancelling arcs and relinking the rest, building a larger set of paths,

If $\sigma_{s}$ receives the confirmation (one search path was successful, i.e. a new augmenting path was found), it broadcasts a reset signal, to prepare the next round. Otherwise, if the search fails, $\sigma_{s}$ receives the backtrack. If there is an untried branch, the round is repeated. Otherwise, $\sigma_{s}$ broadcasts a finalize signal to all cells and the search terminates.

This search algorithm is similar to a classical distributed DFS. Other more efficient distributed DFS algorithms [18] can be considered, but we do not follow this issue here.

\subsection{Distributed BFS-based Strategy}

Our BFS-based algorithms also work in successive rounds:

Each round starts with a set of already established disjoint paths, which is empty at the start of the first round. The source cell, $\sigma_{s}$, broadcasts a "wave", to find new augmenting paths. Current "frontier" cells send out connect signals. The cells which receive and accept these connect signals become the new frontier, by appending themselves at the end of current search paths. The advancing wave periodically sends progress indicators back to the source: (a) connect acknowledgments (at least one search path is still extending) and (b) path confirmations (at least one search path was successful, i.e. at least a new augmenting path was found). While travelling towards the source, each path confirmation reshapes the existing paths and the newly found augmenting path, creating a larger set of paths.

If no progress indicator arrives in the expected time, $\sigma_{s}$ assumes that the search round ends. If at least one search path was successful (at least one augmenting path was found), $\sigma_{s}$ broadcasts a reset signal, which prepares the next round, by resetting all cells (except the target). Otherwise, $\sigma_{s}$ broadcasts a finalize signal to all cells and the search terminates. 
In each round, an intermediate cell, $\sigma_{i}$, can be visited only once. Several search paths may try to visit the same intermediate cell simultaneously, but only one of them succeeds. Figure 4 (a) shows such a scenario: cells 1, 2 and 3 try to connect cell 4 , in the same step; but only cell 1 succeeds, via arc $(1,4)$. This choice operation is further described in Section 6 .

The target cell, $\sigma_{t}$, faces a subtle decision problem. When several search paths arrive, simultaneously or sequentially, $\sigma_{t}$ must quickly decide which augmenting path can be established and which one must be ignored (in the current round). We solve this problem using a branch-cut strategy. Given a search path, $\tau$, its branch ID is the cell ID of its first intermediate cell after the source, taken by $\tau$. Figure 4 (b) shows four potential paths arriving at cell $6: \pi=0.1 .6, \tau_{1}=0.1 .3 .6$, $\tau_{2}=0.1 .5 .6$ and $\tau_{3}=0.2 .4 .6$; their branch IDs are $1,1,1$ and 2 , respectively. Paths $\pi, \tau_{1}$ and $\tau_{2}$ share the same branch ID, 1 , and are incompatible. The following result is straightforward:

Proposition 1. In any search round, search paths which share the same branch ID are incompatible; only one of them can be accepted.

Therefore, the target cell accept or reject decision is based on branch ID. These branch ID operations are further described in Section 6.

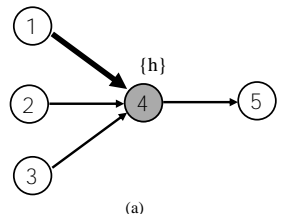

(a)

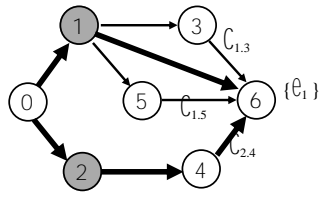

(b)

Fig. 4. BFS challenges. (a) A choice must be made between several search paths connecting the same cell (4), (b) Search paths sharing the same branch ID are incompatible.

\section{P System Rules for Disjoint Paths Using BFS}

The $\mathrm{P}$ system rules for edge- and node-disjoint paths are slightly different, due to the simulated node-splitting approach, but the basic principle is the same. We first discuss the edge-disjoint and then the changes required to cover the node-disjoint.

\subsection{Rules for Edge-disjoint Paths}

\section{Algorithm 1 (P system algorithm for edge-disjoint paths)}

Input: All cells start with the same set of rules and without any topological awareness (they do not even know their local neighbours). All cells start in the 
same initial state. Initially, each cell, $\sigma_{i}$, contains a cell ID object, $\iota_{i}$, which is immutable and used as a promoter. Additionally, the source cell, $\sigma_{s}$, and the target cell, $\sigma_{t}$, are decorated with objects, $a$ and $z$, respectively.

Output: All cells end in the same final state. On completion, all cells are empty, with the following exceptions: (1) The source cell, $\sigma_{s}$, and the target cell, $\sigma_{t}$, are still decorated with objects, $a$ and $z$, respectively; (2) The cells on edgedisjoint paths contain path link objects, for predecessors, $p_{j}$, and for successors, $s_{k}$.

We use the following six states: $S_{0}$, the initial state; $S_{1}$, the quiescent state; $S_{2}$, the frontier state; $S_{3}$, for previous frontier cells; $S_{4}$, the final state; and $S_{5}$, a special state for the target cell.

Initially, all cells are in the initial state, $S_{0}$. When each cell produces a catalystlike object, it enters the quiescent state, $S_{1}$. When cells in $S_{1}$ accept connect signals, they enter the frontier state, $S_{2}$, except the target which changes directly to $S_{5}$. Cells on the frontier send connect signals to neighbors and then change to $S_{3}$, to receive and relay progress indicators. Specifically, the target remains in $S_{5}$, after accepting the first connect signal (because it is always waiting to be connected), until it receives the finalize signal. When the search finishes, all cells transit to the final state, $S_{4}$. Figure 5 shows all state transitions.

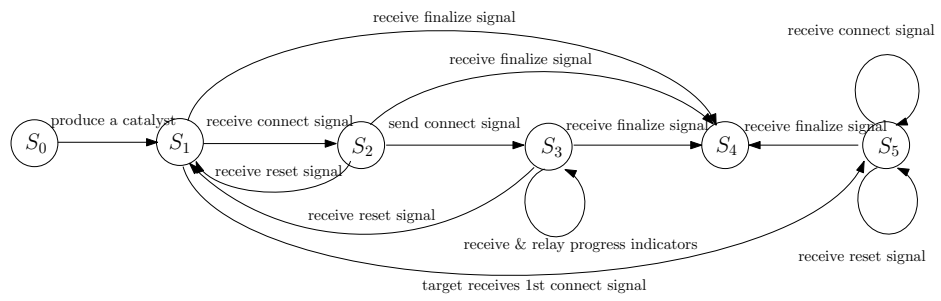

Fig. 5. State-chart of BFS-based algorithm.

We use these symbols to describe our edge-disjoint implementation:

- $a$ indicates the source cell.

- $z$ indicates the target cell.

- $d$ indicates, in the source cell, that an augmenting path was found in the current round (it appears in the source cell).

- $e_{j}$ records, in the target cell, the branch ID of a successful augmenting path (i.e. $\sigma_{j}$ is the first cell after the source, in this augmenting path).

- $c_{s}$ is the connect signal sent by the source cell, $\sigma_{s}$, to its children.

- $c_{j . k}$ is the connect signal sent by an intermediate cell, $\sigma_{k}$, to its children; $j$ is the branch ID.

- $l_{j . k}$ is the connect signal sent by an intermediate cell, $\sigma_{k}$, to its parents; $j$ is the branch ID.

- $r_{j}$ is the connect acknowledgment sent to cell, $\sigma_{j}$. 
- $f_{j . k}$ is the path confirmation of a successful augmenting path, sent by cell $\sigma_{j}$ to cell $\sigma_{k}$.

- $h$ is a catalyst object in each cell.

- $o$ is a signal broadcast by the source cell, $\sigma_{s}$, to make each cell produce one catalyst object.

- $u$ indicates the first intermediate cell after the source, which is produced on receiving the connect signal, $c_{s}$.

- $b$ is the reset signal which starts a new round.

- $g$ is the finalize signal which terminates the search.

- $t_{j}$ indicates that cell $\sigma_{j}$ is a predecessor on a search path (recorded when a cell accepts a connect signal).

- $p_{j}$ is a disjoint path predecessor (recorded when a successful augmenting path is confirmed).

- $s_{j}$ is a disjoint path successor (recorded when a successful augmenting path is confirmed).

- $\quad w, v$ implement a source cell timer to wait for the first response or confirmation.

- $x, y$ implement another source cell timer to wait for the periodically relayed response or confirmation.

We next present the rules and briefly explain them.

0 . Rules in state $S_{1}$ :

$1 S_{0} a \rightarrow_{\min } S_{1} a h(o)_{\downarrow}$

$2 S_{0} o \rightarrow_{\min } S_{1} h(o)_{\downarrow}$

$3 S_{0} o \rightarrow_{\max } S_{1}$

1. Rules in state $S_{1}$ :

$1 S_{1} O \rightarrow_{\max } S_{1}$

$2 S_{1} d \rightarrow_{\max } S_{1}$

$3 S_{1} b \rightarrow_{\max } S_{1}$

$4 S_{1} e_{j} \rightarrow_{\max } S_{1}$

$5 S_{1} g \rightarrow_{\min } S_{4}(g)_{\downarrow}$

$6 S_{1} v \rightarrow_{\max } S_{1}$

$7 S_{1} w \rightarrow_{\max } S_{1}$

$8 S_{1} x \rightarrow_{\max } S_{1}$

$9 S_{1} y \rightarrow_{\max } S_{1}$

$10 S_{1} f_{j . k} \rightarrow_{\max } S_{1}$

$11 S_{1} t_{j} \rightarrow_{\max } S_{1}$

$12 S_{1} r_{j} \rightarrow_{\max } S_{1}$

$13 S_{1} a \rightarrow_{\min } S_{2} a$

$14 S_{1} c_{j} p_{j} \rightarrow_{\min } S_{1} u p_{j}$

$15 S_{1} c_{j . k} p_{k} \rightarrow_{\min } S_{1} p_{k}$

$\left.16 S_{1} z h c_{j . k} \rightarrow_{\min } S_{5} z h p_{k} e_{j}\left(f_{i . k}\right)_{\uparrow}\right|_{\iota_{i}}$

$17 S_{1} z h c_{j} \rightarrow_{\min } S_{5} z h u p_{j}\left(f_{i . j}\right) \uparrow \iota_{\iota_{i}}$

$18 S_{1} h l_{j . k} s_{k} \rightarrow_{\min } S_{2} h t_{k} e_{j} s_{k}\left(r_{k}\right)_{\uparrow}$ 
$19 S_{1} h c_{j} \rightarrow_{\min } S_{2} h_{u t_{j}}\left(r_{j}\right)_{\downarrow}$

$20 S_{1} h c_{j . k} \rightarrow_{\min } S_{2} h t_{k} e_{j}\left(r_{k}\right)_{\uparrow}$

2. Rules in state $S_{2}$ :

$1 S_{2} b \rightarrow_{\min } S_{1}(b)_{\downarrow}$

$2 S_{2} g \rightarrow_{\min } S_{4}(g) \downarrow$

$\left.3 S_{2} a h \rightarrow_{\min } S_{3} a h w\left(c_{i}\right)_{\downarrow}\right|_{\iota_{i}}$

$\left.4 S_{2} h e_{j} \rightarrow_{\min } S_{3} h e_{j}\left(l_{j . i}\right)_{\uparrow}\left(c_{j . i}\right)_{\downarrow}\right|_{\iota_{i}}$

$\left.5 S_{2} h u \rightarrow_{\min } S_{3} h u\left(l_{i . i}\right)_{\uparrow}\left(c_{i . i}\right)_{\downarrow}\right|_{\iota_{i}}$

$6 S_{2} f_{j . k} \rightarrow_{\max } S_{2}$

$7 S_{2} c_{j . k} \rightarrow_{\max } S_{2}$

$8 S_{2} l_{j . k} \rightarrow_{\max } S_{2}$

3. Rules for state $S_{3}$ :

$1 S_{3} b \rightarrow_{\min } S_{1}(b)_{\downarrow}$

$2 S_{3} g \rightarrow_{\min } S_{4}(g)_{\downarrow}$

$3 S_{3}$ axyyf $_{j . i} \rightarrow_{\min } S_{3}$ ads $\left.s_{j} x\right|_{\iota_{i}}$

$4 S_{3}$ axyyr $_{i} \rightarrow_{\min } S_{3}$ ax $\left.\right|_{\iota_{i}}$

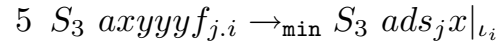

$6 S_{3}$ axyyyr $_{i} \rightarrow_{\min } S_{3}$ ax $\left.\right|_{\iota_{i}}$

$7 S_{3}$ adxyyy $\rightarrow_{\min } S_{1} a(b)_{\downarrow}$

$8 S_{3}$ axyyy $\rightarrow_{\min } S_{4} a(g)_{\downarrow}$

$9 S_{3} a w v v \rightarrow_{\min } S_{4} a(g)_{\downarrow}$

$\left.10 S_{3} a w v f_{j . i} \rightarrow_{\min } S_{3} a d s_{j} x\right|_{\iota_{i}}$

$\left.11 S_{3} a^{a w v r_{i} \rightarrow_{\min }} S_{3} a x\right|_{\iota_{i}}$

$12 S_{3} x \rightarrow_{\min } S_{3} y$

$\left.13 S_{3} t_{j} f_{k . i} \rightarrow_{\min } S_{3} p_{j} s_{k}\left(f_{i . j}\right) \uparrow\right|_{\iota_{i}}$

$\left.14 S_{3} a f_{j . i} \rightarrow_{\min } S_{3} a s_{j}\right|_{\iota_{i}}$

$15 S_{3} p_{j} s_{j} \rightarrow_{\min } S_{3}$

$\left.16 S_{3} r_{i} t_{j} \rightarrow_{\min } S_{3} t_{j}\left(r_{j}\right)_{\uparrow}\right|_{\iota_{i}}$

$17 S_{3} w \rightarrow_{\min } S_{3} w v$

$18 S_{3} r_{j} \rightarrow_{\max } S_{3}$

$19 S_{3} c_{j . k} \rightarrow_{\max } S_{3}$

$20 S_{3} f_{j . k} \rightarrow_{\max } S_{3}$

$21 S_{3} l_{j . k} \rightarrow_{\max } S_{3}$

4. Rules for state $S_{4}$ :

$1 S_{4} g \rightarrow_{\max } S_{4}$

$2 S_{4} e_{j} \rightarrow \max S_{4}$

$3 S_{4} f_{j . k} \rightarrow \max S_{4}$

$4 S_{4} c_{j . k} \rightarrow_{\max } S_{4}$

$5 S_{4} l_{j . k} \rightarrow_{\max } S_{4}$

$6 S_{4} t_{j} \rightarrow_{\max } S_{4}$

$7 S_{4} r_{j} \rightarrow_{\max } S_{4}$

$8 S_{4} w \rightarrow_{\max } S_{4}$ 


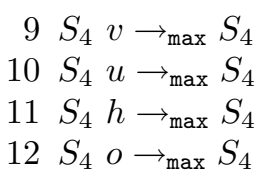

5. Rules for state $S_{5}$ :

$1 S_{5} c_{j} p_{j} \rightarrow_{\min } S_{5} p_{j}$

$\left.2 S_{5} c_{j . k} \rightarrow_{\min } S_{5}\right|_{e_{j}}$

$3 S_{5} c_{j . k} p_{k} \rightarrow_{\min } S_{5} p_{k}$

$\left.4 S_{5} h c_{j . k} \rightarrow_{\min } S_{5} h p_{k} e_{j}\left(f_{i . k}\right) \uparrow\right|_{\iota_{i}}$

$\left.5 S_{5} h c_{j} \rightarrow_{\min } S_{5} h p_{j}\left(f_{i . j}\right)_{\uparrow}\right|_{\iota_{i}}$

$6 S_{5} g \rightarrow_{\max } S_{4}$

$7 S_{5} b \rightarrow_{\max } S_{5}$

$8 S_{5} f_{j . k} \rightarrow_{\max } S_{5}$

$9 S_{5} l_{j . k} \rightarrow_{\max } S_{5}$

$10 S_{5} t_{j} \rightarrow_{\max } S_{5}$

$11 S_{5} r_{j} \rightarrow_{\max } S_{5}$

$12 S_{5} u \rightarrow \max S_{5}$

The following paragraphs outline how these rules are used by each major cell group: the source cell, frontier cells, other intermediate cells and the target cell.

Scripts for the source cell: In the initial state $S_{0}$, the source cell, $\sigma_{s}$, indicated by the special object $a$, starts by broadcasting an object, $o$, to all cells and enters $S_{1}$ (rule 0.1); each receiving cell creates a local catalyst-like object, $h$, and enters $S_{1}$ (rule 0.2 ).

Next, cell $\sigma_{s}$ enters $S_{2}$ (rule 1.13) and starts the search wave via connection requests, $c_{s}$ (rule 2.3). Then, the source cell $\sigma_{s}$ changes to state $S_{3}$ and uses timers to wait (a) one step for the the first progress indicators (rules 3.10, 3.11, 3.17), and (b) two steps for further relayed progress indicators (rules 3.3, 3.4, 3.12). If no progress indicator arrives when the timer overflows, cell $\sigma_{s}$ waits one more step (rules 3.5, 3.6). If still no expected progress indicator arrives, cell $\sigma_{s}$ assumes the round has ended. If an augmenting path was found in the current round, $\sigma_{s}$ broadcasts a reset signal $b$ to reset all cells (except the target $\sigma_{t}$ ) to $S_{1}$ (rule 3.7). Otherwise, $\sigma_{s}$ broadcasts a finalize signal, $g$, which prompts all cells to enter $S_{4}$ (rules 3.8, 3.9).

It is interesting to note why the source cell needs to wait for one more step, even when the timer overflows. An intermediate cell filters connect signals, using rules 1.14-15, which have higher priority than the rules to accept a connect signal, i.e. rules 1.18-20. The rules to accept a connect signal cannot apply in the same step because of the different target states. For example, in Figure 6, path 0.2.4.6.7.9 is found in the first round. In the second round, search paths 0.1 .4 and 0.3 .5 attempt to connect to cell 6 . Cell 6 discards cell 4's connect signal, following the higherpriority rule 1.15 and then, in the next step, accepts cell 5's connect signal, using rule 1.20 . In this case, the source cell needs an extra one-step delay, to receive the 
relayed connect acknowledgment from cell 6 . All unacceptable signals are discarded in one step, so a one-step delay is enough.

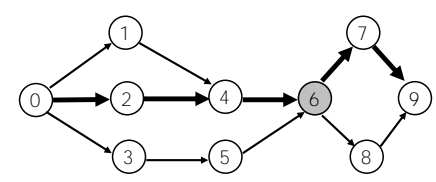

Fig. 6. A particular case requiring a delayed connect acknowledgment.

Scripts for a frontier cell: An intermediate cell, $\sigma_{i}$, if it is unvisited in this round, accepts exactly one connect signal and discards the rest; otherwise, it discards all connect signals. By accepting one connect signal, $\sigma_{i}$ enters $S_{2}$ and becomes a frontier cell to send connect signals. When $\sigma_{i}$ sends its connect signals, the frontier advances.

An intermediate cell, $\sigma_{i}$, may receive connect signals: (a) $c_{s}$, connect signals sent by the source cell, $\sigma_{s}$, to its children; (b) $c_{j . k}$, connect signals sent by a frontier cell, $\sigma_{k}$, to its children; (c) $l_{j . k}$, connect signals sent by a frontier cell, $\sigma_{k}$, to its parents. Received connect signals are checked for acceptability: (a) a $c_{s}$ or $c_{j . k}$ connect signal is acceptable if it does not come from an established path predecessor, which corresponds to a forward operation (rules 1.14, 1.15, 1.19, 1.20); (b) a $l_{j . k}$ connect signal is acceptable if it comes from an established path successor, which corresponds to a push-back operation (rule 1.18).

Cell $\sigma_{i}$ becomes a frontier cell by accepting either: (1) a connect signal, $c_{s}$, from $\sigma_{s}$ (rules 1.14, 1.19), in this case, cell $\sigma_{i}$ (a) generates an $u$, indicating that it is the first intermediate cell on the current search path (the first after cell $\sigma_{s}$ ); (b) records its predecessor on the search path, $\sigma_{s}$, as $t_{s}$; and (c) sends a connect acknowledgment, $r_{s}$, back to cell $\sigma_{s}$; or (2) a connect signal, $c_{j . k}$ or $l_{j . k}$ from $\sigma_{k}$ (rules $1.15,1.18,1.20$ ), in this case, cell $\sigma_{i}$ (a) records the branch ID, $j$, as $e_{j}$; (b) records its predecessor on the search path, $\sigma_{k}$, as $t_{k}$; and (c) sends a connect acknowledgment, $r_{k}$, back to cell $\sigma_{k}$.

Then, as a frontier cell, $\sigma_{i}$ sends connect signals to neighbors and changes to state $S_{3}$ : (1) if cell $\sigma_{i}$ is marked by an $u$ object, it uses its own ID, $i$, as the branch ID to further generate connect signals, $c_{i . i}$ or $l_{i . i}$ (rule 2.5 ); (2) otherwise, $\sigma_{i}$ uses the recorded $e_{j}$ as the branch ID to further generate connect signals, $c_{j . i}$ or $l_{j . i}$ (rule 2.4).

Consider the scenario when several connect signals arrive simultaneously in an unvisited cell, $\sigma_{i}$ (see Figure 4 (a)). Cell $\sigma_{i}$ makes a (conceptually random) choice and selects exactly one of the acceptable connect signals, thus deciding which search path can follow through. To solve this choice problem, we use an object, $h$, which functions like a catalyst [15]. Object $h$ is immediately consumed by the rule which accepts the connect signal, therefore no other connect signal is accepted (rules 1.16-20). Next, the catalyst, $h$, is recreated, but the cell also 
changes its state, thus it cannot accept another connect signal (not in the same search round).

Scripts for other intermediate cell: A previous frontier cell, $\sigma_{i}$, relays progress indicators: connect acknowledgments, $r_{i}$ (rule 3.16) and path confirmations, $f_{k . i}$ (rule 3.13). On receiving path confirmations, $\sigma_{i}$ transforms a temporary path predecessor, $t_{j}$, into an established path predecessor, $p_{j}$, and records the path successors, $s_{k}$. In the next step, cell $\sigma_{i}$ discards matching predecessor and successor objects (i.e. referring to the same cell), e.g., $\sigma_{i}$ may already contain (from a previous round) another predecessor-successor pair, $p_{j^{\prime}}, s_{k^{\prime}}$. If $j=k^{\prime}$, then $p_{j}$ and $s_{k^{\prime}}$ are deleted, as one end of the cancelling arc pair, $(j, i)$ and $(i, j)$; similarly, if $k=j^{\prime}$, then $s_{k}$ and $p_{j^{\prime}}$ are deleted (rule 3.15).

Scripts for the target cell: The target cell, $\sigma_{t}$, accepts either (1) a connect signal from $\sigma_{s}, c_{s}$, if it does not come from an established path predecessor (rules $1.17,5.1,5.5$ ), or $(2)$ a connect signal from a frontier cell $\sigma_{k}, c_{j . k}$ (rules 1.16, 5.2, $5.3,5.4$ ), which indicates the different branch ID (rule 5.2) and does not come from an established path predecessor (rule 5.3). In case (1), cell $\sigma_{t}$ : (a) generates an $u$, indicating that it is the second cell on a search path (the first after cell $\sigma_{s}$ ); (b) records its predecessor on the search path, $\sigma_{s}$, as $p_{s}$; and (c) sends a path confirmation $f_{t . s}$, back to cell $\sigma_{s}$. In case (2), cell $\sigma_{t}$ : (a) records the branch ID, $j$, as $e_{j}$; (b) records its predecessor on the search path, $\sigma_{k}$, as $p_{k}$; and (c) sends a path confirmation, $f_{t . k}$, back to cell $\sigma_{k}$.

This branch-cut strategy is illustrated in Figure 4 (b). It shows an established path, $\pi=0.1 .6$, whose branch ID is recorded as $e_{1}$. Consider the fate of other search paths, $\tau_{1}=0.1 .3 .6, \tau_{2}=0.1 .5 .6$, and $\tau_{3}=0.2 .4 .6$, which attempt to reach the target 6 , later in the same round. $\tau_{1}$ sends the connect signal $c_{1.3}$, which is rejected. $\tau_{2}$ sends the connect signal $c_{1.5}$, which is also rejected. $\tau_{3}$ sends the connect signal $c_{2.4}$, which is accepted. To summarize, in this example round, two augmenting paths are established, $\pi$ and $\tau_{3}$; other attempts are properly ignored.

It is important that recording objects $e_{i}$ are used as promoters, which enable rules, without being consumed [7]. Otherwise, objects $e_{i}$ can be consumed before completing their role; e.g., the rejection of $\tau_{1}$ would consume $e_{1}$ and there would be nothing left to reject $\tau_{2}$.

Example 2. Table 7 shows Algorithm 1 tracing fragments for stages (a), (c) and (e) of Figure 2, illustrating how our $\mathrm{P}$ system solution works. In all stages, each cell, $\sigma_{i}$, contains a promoter object, $\iota_{i}$, as the cell ID; the source cell, $\sigma_{0}$, and the target cell, $\sigma_{7}$, are decorated by objects, $a$ and $z$, respectively. The catalyst object, $h$, remains in each cell after it is produced, until the cell enters the final state, $S_{4}$.

In stage 1(a), the two established paths, 0.1.4.7 and 0.2.5.7, are recorded by the following cell contents: $\sigma_{0}:\left\{s_{1}, s_{2}\right\}, \sigma_{1}:\left\{p_{0}, s_{4}\right\}, \sigma_{2}:\left\{p_{0}, s_{5}\right\}, \sigma_{4}:\left\{p_{1}, s_{7}\right\}$, $\sigma_{5}:\left\{p_{2}, s_{7}\right\}, \sigma_{7}:\left\{p_{4}, p_{5}\right\}$. In the source cell $\sigma_{0}, x y^{3}$ is a timer to wait for the relayed progress indicators, which currently overflows. The object $d$ indicates that an augmenting path was found in the current round, so in the next step, the source cell, $\sigma_{0}$, broadcasts a reset signal to all cells to start a new round. Cells $\sigma_{1}, \sigma_{2}$, and 
Table 7. Algorithm 1 tracing fragments for stages (a), (c) and (e) of Figure 2.

\begin{tabular}{|l|l|l|l|l|}
\hline Stage $\backslash$ Cell & $\sigma_{0}$ & $\sigma_{1}$ & $\sigma_{2}$ & $\sigma_{3}$ \\
\hline $1(\mathrm{a})$ & $S_{3} \iota_{0} a d h s_{1} s_{2} x y^{3}$ & $S_{3} \iota_{1} h p_{0} s_{4} u$ & $S_{3} \iota_{2} h p_{0} s_{5} u$ & $S_{3} \iota_{3} h t_{0} u$ \\
\hline $1(\mathrm{c})$ & $S_{3} \iota_{0} a h s_{1} s_{2} x y$ & $S_{1} \iota_{1} h p_{0} s_{4} u^{2}$ & $S_{3} \iota_{2} e_{3} h p_{0} r_{3} s_{5} t_{5} u^{2}$ & $S_{3} \iota_{3} h r_{3} t_{0} u^{2}$ \\
\hline $1(\mathrm{e})$ & $S_{4} \iota_{0} a s_{1} s_{2} s_{3}$ & $S_{4} \iota_{1} p_{0} s_{4}$ & $S_{4} \iota_{2} p_{0} s_{6}$ & $S_{4} \iota_{3} p_{0} s_{5}$ \\
\hline \hline Stage \Cell & $\sigma_{4}$ & $\sigma_{5}$ & $\sigma_{6}$ & $\sigma_{7}$ \\
\hline $1(\mathrm{a})$ & $S_{3} \iota_{4} e_{1} h p_{1} s_{7}$ & $S_{3} \iota_{5} e_{2} h p_{2} s$ & $S_{3} \iota_{6} e_{2} h t_{2}$ & $S_{5} \iota_{7} e_{1} e_{2} h p_{4} p_{5} z$ \\
\hline $1(\mathrm{c})$ & $S_{1} \iota_{4} f_{7.6} h p_{1} s_{7}$ & $S_{3} \iota_{5} e_{3} f_{7.6} h p_{2} s 7 t_{3}$ & $S_{3} \iota_{6} e_{3} f_{7.6} h t_{2}$ & $S_{5} \iota_{7} e_{1} e_{2} e_{3} h p_{4} p_{5} p_{6} r_{3} z$ \\
\hline $1(\mathrm{e})$ & $S_{4} \iota_{4} p_{1} s_{7}$ & $S_{4} \iota_{5} p_{3} s_{7}$ & $S_{4} \iota_{6} p_{2} s_{7}$ & $S_{4} \iota_{7} p_{4} p_{5} p_{6} z$ \\
\hline
\end{tabular}

$\sigma_{3}$ have objects, $u$, indicating that they are the first intermediate cells after the source, while cells $\sigma_{4}, \sigma_{5}, \sigma_{6}$ contain objects, $e_{j}$, which mean they should include $j$ as the branch ID when sending connect signals. The target cell, $\sigma_{7}$, records the already used branch IDs, $e_{1}$ and $e_{2}$.

In stage $1(\mathrm{c})$, the successful search path 0.3.5.2.6.7 is recorded as: $\sigma_{3}:\left\{t_{0}\right\}$, $\sigma_{5}:\left\{t_{3}\right\}, \sigma_{2}:\left\{t_{5}\right\}, \sigma_{6}:\left\{t_{2}\right\}, \sigma_{7}:\left\{p_{6}\right\}$ (the target records $p_{6}$ directly). The target cell $\sigma_{7}$ also records the branch ID of the newly successful path, $e_{3}$, and sends back a path confirmation $f_{7.6}$ to all its neighbors. In cell $\sigma_{3}$, the objects, $r_{3}$ and $t_{0}$, indicate that the connect acknowledgment needs to be relayed to the source cell $\sigma_{0}$. Thus, in the next step, cell $\sigma_{0}$ receives a connect acknowledgment from cell $\sigma_{3}$ and resets the timer.

In stage 1(e), all cells enter the final state $S_{4}$ and there are three established paths, 0.1.4.7, 0.2.6.7 and 0.3.5.7, which are recorded as: $\sigma_{0}:\left\{s_{1}, s_{2}, s_{3}\right\}, \sigma_{1}$ : $\left\{p_{0}, s_{4}\right\}, \sigma_{2}:\left\{p_{0}, s_{6}\right\}, \sigma_{3}:\left\{p_{0}, s_{5}\right\}, \sigma_{4}:\left\{p_{1}, s_{7}\right\}, \sigma_{5}:\left\{p_{3}, s_{7}\right\}, \sigma_{6}:\left\{p_{2}, s_{7}\right\}$, $\sigma_{7}:\left\{p_{4}, p_{5}, p_{6}\right\}$.

The preceding arguments indicate a bisimulation relation between our BFSbased algorithm and the classical Edmonds and Karp BFS-based algorithm for edge-disjoint paths [4]. The following theorem encapsulates all these arguments:

Theorem 1. When Algorithm 1 terminates, path predecessor and successor objects listed in its output section indicate a maximal cardinality set of edge-disjoint paths.

\subsection{Rules for Node-disjoint Paths}

\section{Algorithm 2 (P system algorithm for node-disjoint paths)}

Input: As in the edge-disjoint paths algorithm of Algorithm 1.

Output: Similar to in the edge-disjoint paths algorithm. However, the predecessor and successor objects indicate node-disjoint paths, instead of edge-disjoint paths.

To simulate node splitting, the node-disjoint version uses additional symbols (as before, rules assume that cell $\sigma_{i}$ is the current cell):

- $m$ indicates that the "entry node is visited".

- $n$ indicates that the "exit node is visited". 
- $q$ indicates that this cell's in-flow and out-flow is one (or, equivalently, that this cell is in an already established or confirmed path).

- $t_{j . k}$ indicates cell $\sigma_{i}$ 's predecessor, $\sigma_{j}$, on a search path, recorded after it receives the connect acknowledgment from cell $\sigma_{i}$ 's successor, $\sigma_{k}$ (before receiving this acknowledgment, $\sigma_{i}$ 's predecessor is temporarily recorded as $t_{j}$.)

- $r_{j . k}$ is a connect acknowledgment sent by cell $\sigma_{j}$ to cell $\sigma_{k}$.

0 . Rules in state $S_{1}$ :

$1 S_{0} a \rightarrow_{\min } S_{1} a h(o)_{\downarrow}$

$2 S_{0} o \rightarrow_{\min } S_{1} h(o)_{\downarrow}$

$3 S_{0} o \rightarrow_{\max } S_{1}$

1. Rules in state $S_{1}$ :

$1 S_{1} o \rightarrow_{\max } S_{1}$

$2 S_{1} d \rightarrow_{\max } S_{1}$

$3 S_{1} b \rightarrow_{\max } S_{1}$

$4 S_{1} e_{j} \rightarrow_{\max } S_{1}$

$5 S_{1} g \rightarrow_{\min } S_{4}(g)_{\downarrow}$

$6 S_{1} v \rightarrow_{\max } S_{1}$

$7 S_{1} w \rightarrow_{\max } S_{1}$

$8 S_{1} u \rightarrow_{\max } S_{1}$

$9 S_{1} m \rightarrow_{\max } S_{1}$

$10 S_{1} n \rightarrow_{\max } S_{1}$

$11 S_{1} f_{j . k} \rightarrow_{\max } S_{1}$

$12 S_{1} t_{j . k} \rightarrow_{\max } S_{1}$

$13 S_{1} t_{j} \rightarrow_{\max } S_{1}$

$14 S_{1} r_{j . k} \rightarrow_{\max } S_{1}$

$15 S_{1} a \rightarrow_{\min } S_{2} a$

$16 S_{1} c_{j} p_{j} \rightarrow_{\min } S_{1} p_{j}$

$17 S_{1} c_{j . k} p_{k} \rightarrow_{\min } S_{1} p_{k}$

$\left.18 S_{1} z h c_{j . k} \rightarrow_{\min } S_{5} z h p_{k} e_{j}\left(f_{i . k}\right)_{\uparrow}\right|_{\iota_{i}}$

$\left.19 S_{1} z h c_{j} \rightarrow_{\min } S_{5} z h p_{j}\left(f_{i . j}\right)_{\uparrow}\right|_{\iota_{i}}$

$\left.20 S_{1} h l_{j . k} s_{k} \rightarrow_{\min } S_{2} h t_{k} e_{j} s_{k} n\left(r_{i . k}\right)\right|_{\iota_{i}}$

$\left.21 S_{1} h c_{j . k} q \rightarrow_{\min } S_{2} h t_{k} e_{j} m q\left(r_{i . k}\right)_{\uparrow}\right|_{\iota_{i}}$

$\left.22 S_{1} h c_{j} \rightarrow_{\min } S_{2} h_{u t_{j}}\left(r_{i . j}\right)_{\uparrow}\right|_{\iota}$

$\left.23 S_{1} h c_{j . k} \rightarrow_{\min } S_{2} h t_{k} e_{j}\left(r_{i . k}\right) \uparrow\right|_{\iota_{i}}$

2. Rules in state $S_{2}$ :

$1 S_{2} b \rightarrow_{\min } S_{1}(b)_{\downarrow}$

$2 S_{2} g \rightarrow_{\min } S_{4}(g)_{\downarrow}$

$\left.3 S_{2} a h \rightarrow_{\min } S_{3} a h w\left(c_{i}\right)_{\downarrow}\right|_{\iota_{i}}$

$\left.4 S_{2} h e_{j} m \rightarrow_{\min } S_{3} h e_{j} m\left(l_{j . i}\right)_{\uparrow}\right|_{\iota_{i}}$

$\left.5 S_{2} h e_{j} n \rightarrow_{\min } S_{3} h e_{j} n\left(l_{j . i}\right)_{\uparrow}\left(c_{j . i}\right)_{\downarrow}\right|_{\iota_{i}}$

$\left.6 S_{2} h e_{j} \rightarrow_{\min } S_{3} h e_{j}\left(l_{j . i}\right)_{\uparrow}\left(c_{j . i}\right)_{\downarrow}\right|_{\iota_{i}}$

$\left.7 S_{2} h u \rightarrow_{\min } S_{3} h u\left(l_{i . i}\right)_{\uparrow}\left(c_{i . i}\right)_{\downarrow}\right|_{\iota_{i}}$ 
$8 S_{2} f_{j . k} \rightarrow_{\max } S_{2}$

$9 S_{2} c_{j . k} \rightarrow_{\max } S_{2}$

$10 S_{2} l_{j . k} \rightarrow_{\max } S_{2}$

3. Rules in state $S_{3}$ :

$1 S_{3} b \rightarrow_{\min } S_{1}(b)_{\downarrow}$

$2 S_{3} g \rightarrow_{\min } S_{4}(g)_{\downarrow}$

$3 S_{3} h m l_{j . k} s_{k} \rightarrow_{\min } S_{3}$ hmnt $\left._{k} e_{j} s_{k}\left(r_{i . k}\right) \uparrow\right|_{\iota_{i}}$

$\left.4 S_{3} h e_{j} m n \rightarrow \min S_{3} h w e_{j}\left(l_{j . i}\right)_{\uparrow}\left(c_{j . i}\right)_{\downarrow}\right|_{\iota_{i}}$

$5 S_{3}$ axyy $_{j . i} \rightarrow_{\min } S_{3}$ ads $\left.s_{j} x\right|_{\iota_{i}}$

$6 S_{3}$ axyyr $_{j . i} \rightarrow_{\min } S_{3}$ ax $\left.\right|_{\iota_{i}}$

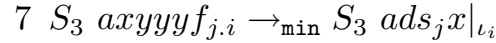

$8 S_{3}$ axyyyr $_{j . i} \rightarrow_{\min } S_{3}$ ax $\left.\right|_{\iota_{i}}$

$9 S_{3}$ adxyyy $\left.\rightarrow_{\min } S_{1} a(b)_{\downarrow}\right|_{\iota_{i}}$

$10 S_{3}$ axyyy $\left.\rightarrow_{\min } S_{4} a(g)_{\downarrow}\right|_{\iota_{i}}$

$11 S_{3}$ awvv $\left.\rightarrow_{\min } S_{4} a(g) \downarrow\right|_{\iota_{i}}$

$\left.12 S_{3} a w v f_{j . i} \rightarrow_{\min } S_{3} a d s_{j} x\right|_{\iota_{i}}$

$13 S_{3}$ awvr $\left._{j . i} \rightarrow_{\min } S_{3} a x\right|_{\iota_{i}}$

$14 S_{3} x \rightarrow_{\min } S_{3} x y$

$\left.15 S_{3} t_{j . k} f_{k . i} \rightarrow_{\min } S_{3} p_{j} s_{k} q\left(f_{i . j}\right) \uparrow\right|_{\iota_{i}}$

$\left.16 S_{3} t_{j} f_{k . i} \rightarrow_{\min } S_{3} p_{j} s_{k} q\left(f_{i, j}\right) \uparrow\right|_{\iota_{i}}$

$\left.17 S_{3} a f_{j . i} \rightarrow_{\min } S_{3} a s_{j}\right|_{\iota_{i}}$

$18 S_{3} p_{j} s_{j} q \rightarrow_{\min } S_{3}$

$\left.19 S_{3} r_{k . i} t_{j . k} \rightarrow_{\min } S_{3} t_{j . k}\left(r_{i . j}\right)_{\uparrow}\right|_{\iota_{i}}$

$\left.20 S_{3} t_{j} r_{k . i} \rightarrow_{\min } S_{3} t_{j . k}\left(r_{i . j}\right) \uparrow\right|_{\iota_{i}}$

$\left.21 S_{3} t_{j . l} r_{k . i} \rightarrow_{\min } S_{3} t_{j . l} t_{j . k}\left(r_{i . j}\right) \uparrow\right|_{\iota_{i}}$

$22 S_{3} w \rightarrow_{\min } S_{3} w v$

$\left.23 S_{3} a r_{j . i} \rightarrow_{\max } S_{3} a\right|_{\iota_{i}}$

$24 S_{3} c_{j . k} \rightarrow_{\max } S_{3}$

$25 S_{3} f_{j . k} \rightarrow_{\max } S_{3}$

$26 S_{3} l_{j . k} \rightarrow_{\max } S_{3}$

4. Rules in state $S_{4}$ :

$1 S_{4} g \rightarrow_{\max } S_{4}$

$2 S_{4} e_{j} \rightarrow_{\max } S_{4}$

$3 S_{4} q \rightarrow_{\max } S_{4}$

$4 S_{4} f_{j . k} \rightarrow_{\max } S_{4}$

$5 S_{4} c_{j . k} \rightarrow_{\max } S_{4}$

$6 S_{4} l_{j . k} \rightarrow_{\max } S_{4}$

$7 S_{4} t_{j . k} \rightarrow_{\max } S_{4}$

$8 S_{4} t_{j} \rightarrow_{\max } S_{4}$

$9 S_{4} r_{j . k} \rightarrow_{\max } S_{4}$

$10 S_{4} w \rightarrow_{\max } S_{4}$

$11 S_{4} v \rightarrow_{\max } S_{4}$

$12 S_{4} u \rightarrow_{\max } S_{4}$ 


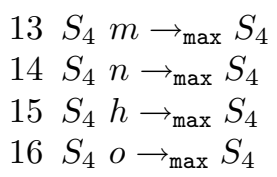

5. Rules in state $S_{5}$ :

$1 S_{5} c_{j} p_{j} \rightarrow_{\min } S_{5} p_{j}$

$\left.2 S_{5} c_{j . k} \rightarrow_{\min } S_{5}\right|_{e_{j}}$

$3 S_{5} c_{j . k} p_{k} \rightarrow_{\min } S_{5} p_{k}$

$\left.4 S_{5} h c_{j . k} \rightarrow_{\min } S_{5} h p_{k} e_{j}\left(f_{i . k}\right) \uparrow\right|_{\iota_{i}}$

$\left.5 S_{5} h c_{j} \rightarrow_{\min } S_{5} h p_{j}\left(f_{i . j}\right)_{\uparrow}\right|_{\iota_{i}}$

$6 S_{5} g \rightarrow_{\max } S_{4}$

$7 S_{5} b \rightarrow_{\max } S_{5}$

$8 S_{5} f_{j . k} \rightarrow_{\max } S_{5}$

$9 S_{5} l_{j . k} \rightarrow_{\max } S_{5}$

$10 S_{5} t_{j . k} \rightarrow_{\max } S_{5}$

$11 S_{5} t_{j} \rightarrow_{\max } S_{5}$

$12 S_{5} r_{j . k} \rightarrow \max S_{5}$

$13 S_{5} u \rightarrow_{\max } S_{5}$

When a cell, $\sigma_{i}$, is first reached by a search path, then both its "entry node" and "exit node" become visited. If this search path is successful, then $\sigma_{i}$ is marked by one object $q$ (rules $3.15,3.16$ ). In a subsequent round, new search paths can visit $\sigma_{i}$ (1) via an incoming arc (forward mode); (2) via an outgoing arc, in the reverse direction (push-back mode) or (3) on both ways. When a search path visits $\sigma_{i}$ via an incoming arc, it marks $\sigma_{i}$ with one object, $m$, indicating a visited entry node (rule 1.21); in this case, the search path can only continue with a push-back (rule 2.4). When a search path visits $\sigma_{i}$ via an outgoing arc, it marks the cell with one object, $n$, indicating a visited exit node (rule 1.20); in this case, the search path continues with all other possible arcs (rule 2.5), i.e. all forward searches and also a push-back on its current in-flow arc. A cell which has a visited entry node is in state $S_{3}$, but it can be later revisited by its exit node. Thus, in $S_{3}$, we provide extra rules to accept and send connect signals (rules $3.3,3.4$ ).

Cell, $\sigma_{i}$, can be visited at most once on each of its entry or exit nodes; but, it can be visited both on its entry and exit nodes, in which case it has two temporary predecessors (which simulate the node-splitting technique). In Figure 8, the search path, 0.4.5.2.1.8.9.3.2.6.7.10, has visited cell 2 twice, once on its "entry" node and again on its "exit" node. Cell 2 has two temporary predecessors, cells 5 and 3, and receives progress indicators from two successors, cells 1 and 6 . Progress indicators relayed by cell 6 must be further relayed to cell 3 and progress indicators relayed by cell 1 must be further relayed to cell 5 . To make the right choice, each cell records matching predecessor-successor pairs, e.g., cell 2 records the pairs $t_{5.1}$ and $t_{3.6}$. For example, when the progress indicator $r_{1.2}$ or $f_{1.2}$ arrives, cell 2 knows to forward it to the correct predecessor, cell 5 . When the progress indicator $r_{6.2}$ or $f_{6.2}$ arrives, cell 2 knows to forward it to the correct predecessor, cell 3. 


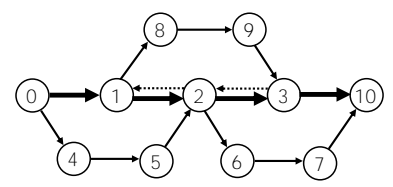

Fig. 8. An example of node-disjoint paths.

The following theorem sums up all these arguments:

Theorem 2. When Algorithm 2 ends, path predecessors and successors objects mentioned in its output section indicate a maximal cardinality set of node-disjoint paths.

\section{Performance of BFS-based Solutions}

Consider a simple $\mathrm{P}$ system with $n$ cells, $m=|\delta|$ arcs, where $f_{e}=$ the maximum number of edge-disjoint paths, $f_{n}=$ the maximum number of node-disjoint paths and $d=$ the outdegree of the source cell. Dinneen at al. show that the DFS-based algorithms for edge- and node-disjoint paths run in $O(m n)$ P steps [3]. A closer inspection, not detailed here, shows that this upper bound can be improved.

Theorem 3. The DFS-based algorithms run in $O(m d) P$ steps, in both the edgeand node-disjoint cases.

We show that our algorithms run asymptotically faster $\left(f_{e}, f_{n} \leq d\right)$ :

Theorem 4. Our BFS-based algorithms run in at most $B(m, f)=(3 m+5) f+$ $4 m+6$ P steps, i.e. $O(m f)$, where $f=f_{e}$, in the edge-disjoint case, and $f=f_{n}$, in the node-disjoint case.

Proof. 1. Initially, the source cell broadcasts a "catalyst" in one step.

2. Then, the algorithm repeatedly searches augmenting paths. First, consider the rounds where augmenting paths are found. In each round, each cell on the search path takes two steps to proceed, i.e. one step to accept a signal and one more step to send connect signals. Each search path spans at most $m$ arcs, thus it takes at most $2 m$ steps to reach its end (with or without reaching the target). All search paths in a round proceed in parallel. After the last augmenting path in a round was found, it takes at most $m$ steps to confirm to the source. After receiving the last confirmation signal, the source cell waits four steps (to ensure that it is the last) and then takes one step to broadcast a reset signal. Therefore, each round, where augmenting paths are found, takes at most $3 m+5$ steps. At least one augmenting path is found in each round, so the total number of search rounds is at most $f$. 
3. Next, consider the last search round, where no more augmenting paths are found. This case is similar, but not identical, to the preceding case. Each cell on the search path takes two steps to proceed, so it takes at most $2 m$ steps to search augmenting paths. The connect acknowledgment from the end cell of the search path takes at most $m$ steps to arrive at the source. The source waits for three or four steps for the time-out: three steps, if it does not receive any progress indicators; and four steps, otherwise. Then, the source cell broadcast a finalize signal, which takes at most $m$ steps to reach all cells.

4. Finally, all cells take one final step, to clear all irrelevant objects, and the algorithm terminates.

To summarize, the algorithm runs in at most $(3 m+5) f+4 m+6$ steps and its asymptotic runtime complexity is $O(m f)$.

Table 9 compares the asymptotic complexity of our BFS-based algorithms against some well-known maximum flow BFS-based algorithms. Our BFS-based algorithms are faster, because they leverage the potentially unbounded parallelism inherent in $\mathrm{P}$ systems.

Table 9. Asymptotic worst-case complexity: classical BFS-based algorithms (steps), P system DFS-based algorithms [3] (P steps) and our P system BFS-based algorithms (P steps).

\begin{tabular}{|l|l|}
\hline Edmonds-Karp [4] & $O\left(m^{2} n\right)$ steps \\
\hline Dinic [2] & $O\left(m n^{2}\right)$ steps \\
\hline Goldberg and Tarjan [6] & $O\left(n m \log n^{2} / m\right)$ steps \\
\hline P System DFS-based [3] & $O(m d)$ P steps \\
\hline P System BFS-based [here] & $O(m f)$ P steps \\
\hline
\end{tabular}

Theorem 4 indicatess the worst-case upper bound, not the typical case. A typical search path does not use all $m$ arcs. Also, the algorithm frequently finds more than one augmenting paths in the same search round, thus the number of rounds is typically much smaller than $f$. Therefore, the average runtime is probably much less than than the upper bound indicated by Theorem 4. Empirical results, obtained with our in-house simulator (still under development) support this observation.

Table 11, empirically compares the performance of our BFS-based algorithms against the DFS-based algorithms [3], for the scenarios of Figure 10. The empirical results show that BFS-based algorithms take fewer P steps than DFS-based algorithms. The performance is, as expected, influenced by the number of nodes and the density of the digraph. Typically, the ratio of BFS:DFS decreases even more, with the complexity of the digraph. We conclude that, the empirical complexity is substantially smaller than the asymptotic worst-case complexity indicated by Theorem 4. 

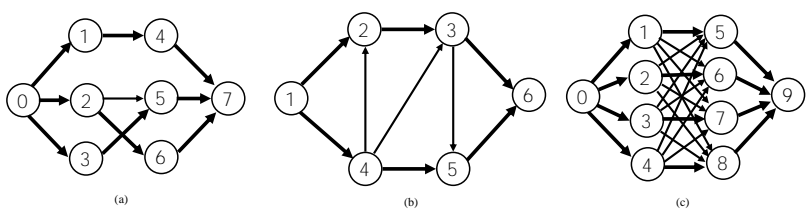

Fig. 10. Empirical tests of BFS-based and DFS-based algorithms.

Table 11. Empirical complexity of BFS-based and DFS-based algorithms (P steps).

\begin{tabular}{|l|l|l|l|l|l|l|l|}
\hline $\begin{array}{l}\text { Test } \\
\text { Case }\end{array}$ & $m$ & $f=f_{e}, f_{n}$ & $B(m, f)$ & EFS Empirical Complexity & DFS Empirical Complexity \\
\hline (a) & 10 & 3 & 151 & 44 & 45 & 63 & 62 \\
\hline (b) & 9 & 2 & 106 & 24 & 24 & 61 & 59 \\
\hline (c) & 24 & 4 & 410 & 66 & 75 & 241 & 194 \\
\hline
\end{tabular}

\section{Conclusions}

We proposed the first BFS-based P system solutions for the edge- and node-disjoint paths problems. As expected, because of potentially unlimited parallelism inherent in $\mathrm{P}$ systems, our $\mathrm{P}$ system algorithms compare favourably with the traditional BFS-based algorithms. Empirical results show that, in terms of P steps, our BFSbased algorithms outperform the previously introduced DFS-based algorithms [3].

Several interesting questions and directions remain open. Can we solve this problem using a restricted $\mathrm{P}$ system without states, without sacrificing the current descriptive and performance complexity? What is the average complexity of our BFS-based algorithms? How much can we speedup the existing DFS-based algorithms, by use more efficient distributed DFS algorithms? An interesting avenue is to investigate a limited BFS design, in fact, a mixed BFS-DFS solution, which combines the advantages of both BFS and DFS. Finally, another direction is to investigate disjoint paths solutions on $\mathrm{P}$ systems with asynchronous semantics, where additional speedup is expected.

\section{Acknowledgments}

The authors wish to thank Tudor Balanescu, Michael J. Dinneen, Yun-Bum Kim, John Morris and three anonymous reviewers, for valuable comments and feedback that helped us improve the paper.

\section{References}

1. Cormen, T.H., Stein, C., Rivest, R.L., Leiserson, C.E.: Introduction to Algorithms. The MIT Press, 3rd edn. (2009) 
2. Dinic, E.A.: Algorithm for solution of a problem of maximum flow in a network with power estimation. Soviet Math. Dokl., 11, 1277-1280 (1970)

3. Dinneen, M.J., Kim, Y.B., Nicolescu, R.: Edge- and node-disjoint paths in P systems. Electronic Proc. in TCS, 40, 121-141 (2010)

4. Edmonds, J., Karp, R.M.: Theoretical improvements in algorithmic efficiency for network flow problems. J. ACM, 19(2), 248-264 (1972)

5. Ford, L.R., Jr., Fulkerson, D.R.: Maximal flow through a network. Canadian Journal of Mathematics, 8, 399-404 (1956)

6. Goldberg, A.V., Tarjan, R.E.: A new approach to the maximum flow problem. J. ACM, 35(4), 921-940 (1988)

7. Ionescu, M., Sburlan, D.: On p systems with promoters/inhibitors. J. Universal Computer Science, 10(5), 581-599 (2004)

8. Kozen, D.C.: The Design and Analysis of Algorithms. Springer, New York, NY, USA (1991)

9. Lynch, N.A.: Distributed Algorithms. Morgan Kaufmann Publishers Inc., San Francisco, CA, USA (1996)

10. Martín-Vide, C., Păun, G., Pazos, J., Rodríguez-Patón, A.: Tissue P systems. Theor. Comput. Sci. 296(2), 295-326 (2003)

11. Nicolescu, R., Dinneen, M.J., Kim, Y.B.: Structured modelling with hyperdag P systems: Part A. Report CDMTCS-342, Centre for Discrete Mathematics and Theoretical Computer Science, The University of Auckland, Auckland, New Zealand (December 2008), http://www.cs.auckland.ac.nz/CDMTCS/ researchreports/342hyperdagA.pdf

12. Nicolescu, R., Dinneen, M.J., Kim, Y.B.: Structured modelling with hyperdag P systems: Part B. Report CDMTCS-373, Centre for Discrete Mathematics and Theoretical Computer Science, The University of Auckland, Auckland, New Zealand (October 2009), http://www.cs.auckland.ac.nz/CDMTCS//researchreports/373hP_B.pdf

13. Nicolescu, R., Dinneen, M.J., Kim, Y.B.: Towards structured modelling with hyperdag P systems. Intern. J. Computers, Comm. and Control, 2, 209-222 (2010)

14. Păun, G.: Membrane Computing: An Introduction. Springer, New York, Inc., Secaucus, NJ, USA (2002)

15. Păun, G.: Introduction to membrane computing. In: Ciobanu, G., Pérez-Jiménez, M.J., Păun, G. (eds.) Applications of Membrane Computing, pp. 1-42. Natural Computing Series, Springer (2006)

16. Păun, G., Centre, T., Science, C.: Computing with membranes. J. Computer and System Sciences, 61, 108-143 (1998)

17. Seo, D., Thottethodi, M.: Disjoint-path routing: Efficient communication for streaming applications. In: IPDPS. pp. 1-12. IEEE (2009)

18. Tel, G.: Introduction to Distributed Algorithms. Cambridge Univ. Press (2000) 
